\title{
Inter-Network Resource Sharing in IEEE 802.22 Networks
}

\author{
Carla Passiatore, Pietro Camarda \\ DEE-Politecnico di Bari v. Orabona, Bari, Italy \\ Email: \{c.passiatore, camarda\}@poliba.it
}

Received February 20, 2012; revised March 18, 2012; accepted April 10, 2012

\begin{abstract}
IEEE 802.22 is the first worldwide standard for Cognitive Networks (CNs) that exploits unused spectrum of the television broadcast service. An IEEE 802.22 network is also called Wireless Regional Area Network (WRAN). An open issue in cognitive 802.22 networks is represented by the resource distribution among WRANs. In this paper a protocol for radio resource management for CNs in a multichannel environment is presented and analysed. In particular, the contribution of this work is a resource sharing method to schedule the WRAN access to the available channels in a community made by two o more coexisting WRANs. The method adapts to the continuous changes of the spectrum availability due to necessity of vacating a channel in case of the incumbent primary users. Moreover, the introduced allocation scheme allows to divide the available band in a proportional way to the cognitive user spectrum demands, taking into account the issue of spatial diversity, i.e. the case where the channel coverage area is not uniform. The effectiveness of the proposed multichannel scheme is proved through simulations. The results compared favorably with other methods already known in literature and show that the proposed algorithm optimize the spectral efficiency, keeping high fairness as demonstrated computing the Jain's index*.
\end{abstract}

Keywords: Cognitive Radio; Resource Sharing Protocol; IEEE 802.22

\section{Introduction}

The ever increasing number of users of wireless networks, with their demand of sophisticated services have as consequence the pressing request of spectrum resource. The current spectrum assignment policy allocates to licensed users the frequency bands exclusively for specific services. A recent investigation on the spectrum utilization made by the Federal Communications Commission (FCC) has demonstrated an inefficient usage of the spectrum. The band is not exploited with time continuity but there are temporal intervals during which the users are idle and the transmission channel is unused. As an example, below some spectrum occupancy measurements are reported, [3]: in New York City the maximum total spectrum occupancy is only $13.1 \%$ from $30 \mathrm{MHz}$ to 3 $\mathrm{GHz}$; in Washington the band occupancy is less than $35 \%$ for the radio spectrum below $3 \mathrm{GHz}$. The problem of the inefficient resource usage is related to the fixed spectrum assignment policy.

The concept of cognitive radio (CR) has been introduced to resolve the issue of spectrum scarcity and its inefficient usage. Cognitive radios are particular types of devices that can sense the environment and autonomously

${ }^{*}$ This work extends and generalizes the previous two conference papers [1] and [2]. adapt its operating characteristics. The network which accommodates CRs is consequently called Cognitive Network (CN). The core issue of a $\mathrm{CN}$ is based on the definition of spectrum holes and the distinction between primary and secondary users. Primary users (PUs) are licensed users who have the right to employ certain channels whenever they need. Secondary users (SUs) are unlicensed users who sense the spectrum, and are allowed to transmit on the available frequencies with the condition of not cause any harmful interference to the PUs [4].

A spectrum band is underused if it can accommodate further transmissions without interfering with other users. The region of space-time-frequency in which a secondary use is possible is called spectrum hole or white space [5].

IEEE 802.22 is a standard for Wireless Regional Area Network (WRAN) using in an opportunistic way the white spaces in the TV frequency spectrum, exploiting cognitive radio techniques.

Since the spectrum is already assigned, one of the most important challenge is to share the licensed spectrum without interfering with the transmission of the PUs. Spectrum sharing techniques could be classified into two types intra-network or inter-network spectrum sharing. Intra-network spectrum sharing is the activity to share the resources spectrum inside a CR network among its 
SUs. It is referred to inter-network for the channel sharing among multiple coexisting CR networks.

IEEE 802.22 standard provides two types of inter-BS dynamic resource sharing mechanisms, namely Resource Renting and On-Demand Spectrum Contention (ODSC), which face the self coexistence issue. The former mechanism is a nonexclusive spectrum sharing scheme that allows BSs to share one or more channels by selectively renting candidate (surplus) channels of neighbouring cells. The latter mechanism is an exclusive spectrum sharing scheme which is based on contention. The BS which needs spectrum, contends for candidate channels of neighbouring BSs. Bian proposed in [6] a coexistence-aware spectrum sharing protocol which represents a trade-off between two methods: incumbent coexistence and self coexistence. In fact, it switches between exclusive and non-exclusive spectrum sharing to minimize self interference keeping overhead under control.

Current research issues on cognitive networks are focused also on the development of algorithms for channel sharing among several WRANs. But while in the case where the channel number is higher than the WRAN number some solutions have been presented in literature $[7,8]$, the situation where the channel number is smaller than the WRAN number has not been studied enough. In [7], a resource allocation solution in case of available channel number higher than the WRAN number, namely Dynamic Frequency Hopping (DFH), is exposed. The data transmission is performed in parallel with spectrum sensing without interruptions, by the use of simultaneous sensing and data transmission. DFH mode requires two channels for each WRAN, one for sensing and the other for transmission. Consequently, for $N$ WRANs, $2 N$ channels are needed. In [7] a solution is introduced to allow the DFH mode using only $N+1$ channels for $N$ WRANs.

To increase the spectral efficiency avoiding interference among different WRANs the theory of coloured graph was introduced in literature $[9,10]$. The graph is obtained starting from the topology of the network; each $\mathrm{BS}$ in the region is denoted by a vertex, and each vertex is connected to another if the corresponding WRANs are overlapped. Each vertex is coloured, but if there is a connection between any two vertices, those two vertices cannot have the same color. The chromatic number of the graph is the minimum number of distinct colors needed to color the graph. If $x$ is the chromatic number of a graph, it is said $x$ colorable. Since to each color corresponds a channel, the chromatic number is the minimum number of distinct channels required for the simultaneous transmission of all WRANs without causing interference among them. In [10] a spectrum allocation policy based on graph theory is proposed. A possible way to share the band is the Max-min fairness criterion, [11]. The drawback of these methods is that it is not fair. The same amount of band is assigned to the WRANs characterized by the same color in the graph, even if they do not have the same band requests. Moreover the Max-Min fairness criterion gives a priority to the smaller flows, in the sense that the WRANs with low demands will be completely satisfied, unlike WRANs with high demands. Furthermore, these policy assume that the channels are available for all the WRANs. The size of a 802.22 WRAN can reach up to $100 \mathrm{Km}$, then it is possible that the WRANs have different sensing results.

In this paper we propose a spectrum allocation algorithm which coordinates the transmission of different overlapped 802.22 WRANs, determining a fair spectrum scheduling method among the coexisting networks. Our contribution in this context is a centralized internetwork resource sharing (CIRS) scheme which unites the characteristic of spectrum efficiency with fairness criteria. Moreover it is a dynamic scheme, which is able to adapt to the continuous changes of the channel availability which are proper to a $\mathrm{CN}$. The introduced approach is suited to any scenario, regardless by the number of WRANs in the network and the amount of available resources.

The paper is structured as follows. In Section 2 an overview on the IEEE 802.22 standard is presented. In Section 3 the novel protocol is introduced describing the algorithm exploited for the channel allocation process; the assignment process is explained with the help of an example. In Section 4 the performance evaluations of the protocol are shown, moreover the protocol accomplishment is compared with the performances of other methods already known in literature. Section 5 draws the conclusions.

\section{Overview on IEEE 802.22 Networks}

The IEEE 802.22 standard is the first standard for CRs which exploits in an opportunistic way the idle or underutilized spectrum in the TV broadcast band $[7,12]$.

As for a common $\mathrm{CN}$ in a 802.22 network there are SUs which are allowed to employ licensed bands with the condition not to interfere with PUs. In fact, if SUs sense incumbents ready to transmit they have to vacate the channel. The feature of a 802.22 network is that the primary users are TV transmitters and wireless microphones.

The 802.22 network is a centralized structure composed by a Base Station (BS) and Customer Premise Equipments (CPEs). Radio resource management is designed to grow the network efficiency [13]. Inside a 802.22 WRAN the intranetwork spectrum sharing process is managed by the BS.

The BS, according to the spectrum availability and the policy of channel sharing, decides which is the CPE of its network which is allowed to accede to the medium. The 
IEEE 802.22 WRAN is a network with infrastructure, where the BS covers a wide area, typically from $30 \mathrm{~km}$ to $100 \mathrm{~km}$, according to the power, range is $33 \mathrm{Km}$ at 4 Watts. A typical 802.22 network is illustrated in Figure 1.

The communication of the BS with its CPEs happens in both the directions, from the BS to the CPE, which is named downstream, and in the opposite way when a CPE responds back to the $\mathrm{BS}$ in the upstream direction. The 802.22 frame is divided into two parts: downstream (DS) subframe and upstream (US) subframe. The first is used to transmit information from the BS to CPEs; while in the second the communication direction is reversed. DS and US subframes are separated by a transmit transition gap. The DS consists of a single packet burst to a given CPE while US consists of multiple packets from several CPEs [14]. The BS coordinates as well as the transmission also the sensing operations. At the aim to decrease the probability of false alarm in an 802.22 network the sensing is distributed generally. Based on the feedback received by the CPEs, the $\mathrm{BS}$ decides which decision has to be taken. IEEE 802.22 is a time slotted protocol [15]; the operations are spread in a slotted structure composed by frames and superframes. A 802.22 superframe is composed by 16 frames. During each MAC frame, the BS has the responsibility to manage the upstream and downstream operations, which may include ordinary data communication, measurement activities, coexistence procedures, and so on. The duration of a frame is $10 \mathrm{~ms}$, than a superframe is $160 \mathrm{~ms}$ [16]. The superframe shall start with a PHY superframe preamble, followed by the first frame preamble, the Superframe Control Header and finally the frame payload, as shown in Figure 2.

On the other side the frames of the superframe are only composed by a preamble followed by the payload. For this reason the first frame payload is reduced compared to other frames. The $\mathrm{SCH}$ is transmitted by the BS at the beginning of the superframe on the operating channel. So all the CPEs tuned to that channel can synchronize with the $\mathrm{BS}$, which manages their operations.

\section{Spectrum Allocation Process}

In this Section the introduced algorithm is explained referring to an example. The scenario is composed by cognitive 802.22 networks, which form a community. In the community a BS of a WRAN is elected as coordinator, while the other WRANs are simple memberships. To elect the leader the algorithm introduced in [17] is proposed. It is considered ideal because it is appropriate for large groups. The method uses a hierarchical structure, introducing a solution in case of the crash of the coordinator. In the introduced method the leader will be the responsible of the membership channel access. The transmissions may be coordinated in such a way to avoid

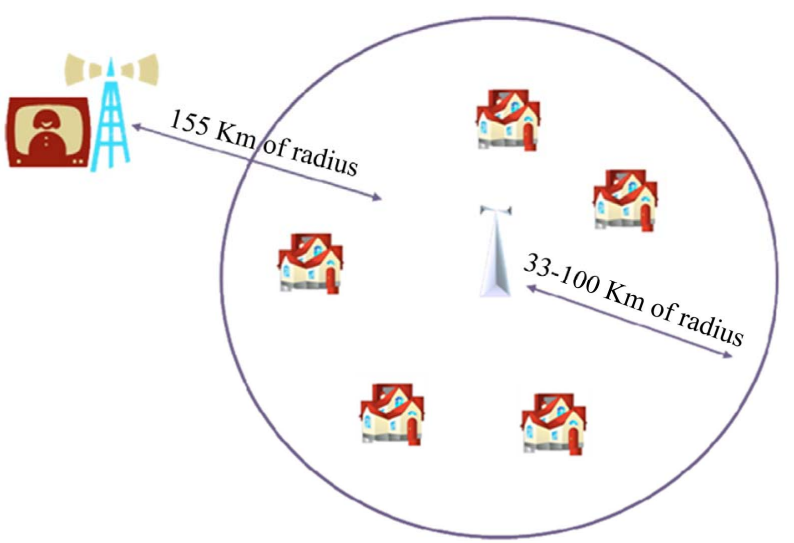

Figure 1. An example of 802.22 network.

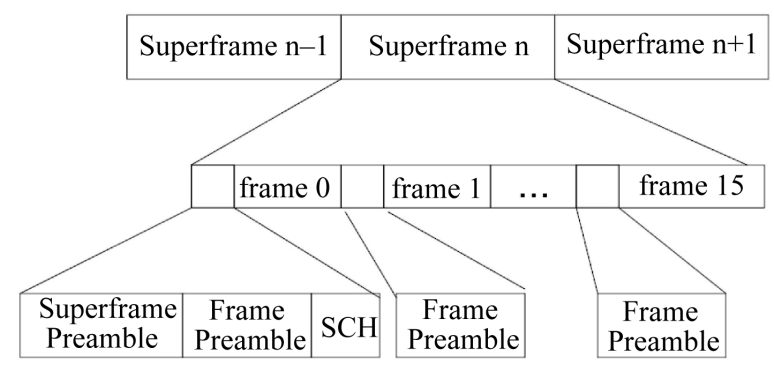

Figure 2. An example of 802.22 superframe.

harmful interference among the WRANs. The example exposed above is referred to a scenario where there are overlapped WRANs as shown in Figure 3.

The method is based on the interaction among the leader and the other memberships of the community which exchange information each other. For the inter-network communication the 802.22 standard addresses to the Coexistence Beacon Protocol (CBP) [18]. In particular the BSs communicate to the leader: 1) its neighbourhood and overlapping WRANs, 2) resource request, 3) available channels. We assume that if the BS hears a channel it is available for all the CPEs of the WRAN. The coordinator exploits these data for resource sharing optimization. The main activities of the community members are three: sensing, communication with the leader, transmission according to the coordinator decisions.

In a IEEE 802.22 network the sensing operation must be done periodically, with a period no larger then two second [9]. For this reason the exchanged information and the transmission should last no more than $2 \mathrm{~s}$.

During the sensing period each WRAN performs the sensing activity finding available band and verifying that channels marked as unused are still available. After this, the BSs convey their sensing results to the coordinator, which works as a central node collecting topology information and WRANs' spectrum requests. According to these data the coordinator decides the WRANs which are allowed to transmit and the respective channels to be 


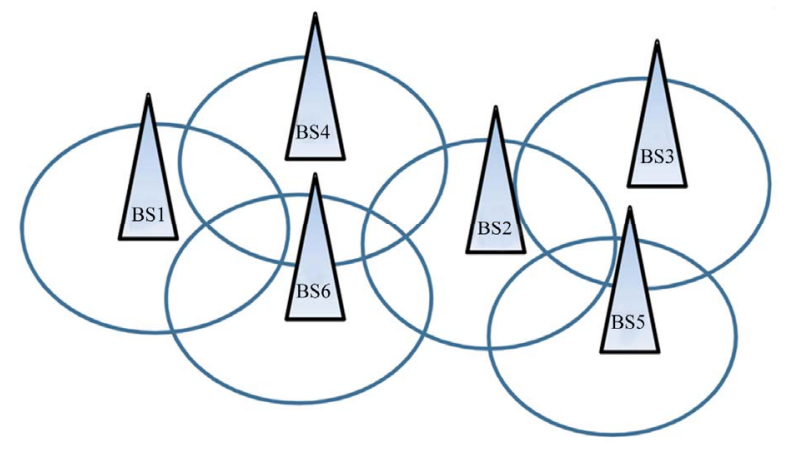

Figure 3. Example of overlapped WRAN configuration.

occupied. At the end of this period the coordinator dispenses the channel access map to the community members. Then in the transmission period each WRAN BS manages the transmission of its CPEs according to the 802.22 standard and the channel access map.

\subsection{Channel Allocation}

The features of the introduced assignment policy are:

- Reuse of the spectral frequency;

- Resource sharing with regards to the spatial diversity, i.e. the availability of some channels only for some WRANs;

- Resource sharing according to the time diversity, i.e. the variance over time of the channel availability;

- Channel distribution according to the WRAN spectrum demands.

To satisfy the firsts two points the coordinator needs to evaluate for each channel, the group of WRANs which are able to transmit simultaneously without causing interference. The coordinator elaborates the access plan to the community resources and dispenses the channel access map to each WRAN. The allocation process involves a maximum of 12 superframes; since the maximum time that a channel may be occupied by a secondary user is $2 \mathrm{~s}$ [12], and the superframe duration is $160 \mathrm{~ms}$ [16]. After this period a new sensing is necessary, and the coordinator has to implement again the channel allocation process according to the new topology information.

To collect topology information the coordinator use a overlay table, while for the available resources and the special diversity a channel table is exploited. The overlay table and channel table have to be updated as soon as new information is received.

The overlay table is a square matrix, with size $N$, where $N$ is the total number of WRANs. The $(i, j)$ element of the matrix is equal to 1 if the WRAN $i$ and the WRAN $j$ are overlapped. While the channel table has the number of rows equal to the number of WRANs in the community, and a number of columns equal to the number of channels. The $(i, j)$ element of the channel table is equal to 1 if the channelj is available for the WRAN $i$.
Referring to the topology of Figure 3, Table 1 shows the overlay table. For reasons of compactness in Table 1 and in the other tables of this paper the notation $W 1$ stands for WRAN1, and so on.

The channel table is created on the basis of the received sensing information. Let us suppose to have three available channels with different coverage area, which allow to build up the channel table as shown in Table 2. In particular, we supposed that channelA is available only for WRAN1, WRAN4 and WRAN6, channelB is available only for WRAN2, WRAN3 and WRAN5, while the third channel covers all the network.

Note that the amount of information exchanged among WRANs and the coordinator is exiguous; each WRAN has to communicate only the channel availability, the WRANs overlapped with itself and its requests. The concept of request will be clarified in the following.

According to the contents of the Tables 1 and 2, the coordinator is able to elaborate the sets of WRANs which could transmit simultaneously without interfering each other. In the following these combinations are called clusters. The principle used to compute the clusters is that if two WRANs are overlapped among them, they cannot use the same channel simultaneously.

The procedure to compute the clusters is:

- For each channel the coordinator has to examine which are the WRANs which can use it; i.e. for each channel table column the coordinator checks the elements marked with 1.

- Considering only this WRAN set the coordinator computes the non-overlapped WRAN groups.

In the exposed example WRAN1, WRAN4, and WRAN6 are overlapped among them, then only one of these

Table 1. Overlay table.

\begin{tabular}{ccccccc}
\hline & $W 1$ & $W 2$ & $W 3$ & $W 4$ & $W 5$ & $W 6$ \\
\hline$W 1$ & 1 & 0 & 0 & 1 & 0 & 1 \\
$W 2$ & 0 & 1 & 1 & 1 & 1 & 1 \\
$W 3$ & 0 & 1 & 1 & 0 & 1 & 0 \\
$W 4$ & 1 & 1 & 0 & 1 & 0 & 1 \\
$W 5$ & 0 & 1 & 1 & 0 & 1 & 0 \\
$W 6$ & 1 & 1 & 0 & 1 & 0 & 1 \\
\hline
\end{tabular}

Table 2. Channel table.

\begin{tabular}{cccc}
\hline & ChannelA & ChannelB & ChannelC \\
\hline$W 1$ & 1 & 0 & 1 \\
$W 2$ & 0 & 1 & 1 \\
$W 3$ & 0 & 1 & 1 \\
$W 4$ & 1 & 0 & 1 \\
$W 5$ & 0 & 1 & 1 \\
$W 6$ & 1 & 0 & 1 \\
\hline
\end{tabular}


WRANs at a time can occupy the channelA. While more WRANs can transmit simultaneously using the channel $C$ without interfering each other. In Table $\mathbf{3}$ are shown all the possible channel assignments. Precisely, each column of the table is referred to a specific channel. Each row shows the possible combinations of WRANs which can transmit simultaneously, without interfering each other, occupying the same channel, which is indicated on the top of the column.

The coordinator creates the access map, which indicates the WRANs able to transmit at a given moment. The map is created selecting for each channel a single WRAN cluster which are allowed occupy it for a superframe. The choice is taken also according to the band requests. Specifically, the goal is to assign the resources proportionally the requests, taking into account the spatial diversity. In the following how to reach this objective is exposed.

The proposed resource sharing algorithm allows the best distribution among WRANs of the superframes. The distribution takes into account two different aspects: the total number of assigned superframes and the fairness of the allocation. The target is to take the fairest choice among the ones which maximize the total number of assigned superframes. Below the policy is explained and after that an example is introduced to clarify the mechanism.

Each BS estimates the total amount of data which its CPEs need to transmit. The BSs communicate to the leader this information during the phase of communication with the coordinator. The coordinator for each WRANi estimates request ${ }_{i}$, which is the number of superframes requested by WRANi; request $t_{i}$ is evaluated according to the channel bandwidth and the amount of data to be transmitted by WRANi.

According to the requests the coordinator computes the transmission probability $p_{i}$, by using the following formula:

$$
p_{i}=\frac{\text { request }_{i}}{\sum_{j=1}^{N} \text { request }_{j}}
$$

where $N$ is the number of WRANs in the community.

Intuitively, $p_{\mathrm{i}}$ represents the expected probability that WRANi shares the resource, that is the expected portion of band divided by WRANi during the transmission period. All the $p_{i} \mathrm{~s}$ are included in the probability vector, namely $p$, where the $i^{\text {th }}$ element is the transmission probability of the WRANi. The values of $p_{i}$ can be different among the WRANs because related to the resource request of each of them, but the sum of all $p_{i} \mathrm{~s}$ is equal to one.

In the exposed case the WRAN clusters which can transmit simultaneously without producing interference
Table 3. Available channel table.

\begin{tabular}{ccc}
\hline ChannelA & ChannelA & ChannelA \\
\hline W1 & W2 & W1 - W2 \\
W4 & W3 & W1 - W3 \\
W6 & W5 & W1 - W5 \\
- & - & W3 - W4 \\
- & - & W3 - W6 \\
- & - & W4 - W5 \\
- & - & W5 - W6 \\
\hline
\end{tabular}

are illustrated in Table 3. As an example in Table 3, 7 different combinations are individuated for channelC, namely (W1, W2), (W1, W3), (W1, W5), (W3, W4), (W3, W6), (W4, W5), (W5, W6). To define the channelC access map the coordinator must choose one of the above combination of WRANs to assign the resource.

To explain the algorithm used to choose the cluster for transmission, we have to introduce the state vector, namely $s_{-} v$, which is a vector, of $N$ elements, where the $i^{\text {th }}$-element represents the total number of superframes assigned to the WRANi. As an example, vector [3, 3, 0, 0 , $0,0]$ represents the state where 3 superframes have been assigned to WRAN1 and WRAN2, while the other WRANs do not have resources. The $s \_v$ is updated superframe by superframe.

The criteria introduced in order to determine the best final $s \_v$ is the one which maximizes $y$, where:

$$
y=\sum_{i=1}^{N} p_{i} \ln \left(n_{i}+1\right)
$$

where $n_{i}$ is the number of superframes assigned to WRANi, with $\mathrm{n}_{i} \geq 0$. Note that at the beginning of the assignment process the $s \_v$ is a null vector with length equal to the number of the WRANs.

An intuitive explanation, to justify how the function $y$ was built is reported in the subsection Consideration on the function $y$.

Note that at the begin all the $\mathrm{n}_{\mathrm{i}} \mathrm{s}$ are equal to 0 ; the $s \_v$ of the scenario in the Figure 3 is $s_{-} v=[0,0,0,0,0,0]$. Every time that the coordinator gives to the WRAN $i$ the possibility to transmit during a superframe using a channel, the $i^{\text {th }}$-element of the $s_{-} v$ is incremented of a unit. For each available channel and for each superframe, the coordinator has to choose among the combinations of Table 3 the one that gives the $s \_v$ which maximizes the function of Equation (2). Starting from the channel which covers a lower area, the coordinator evaluates all the possible values of $s \_v$ which could be chosen according to the cluster of Table 3. For each entry of Table 3, the result of Equation (2) must be calculated, and finally the state which returns as result the highest value of y must be chosen. Then the $s \_v$ is updated. The procedure is re- 
peated for each available channel. Moreover, we suppose that each $\mathrm{BS}$ is able to manage a maximum of $M$ channels at time; then the coordinator cannot assign more than $M$ channels to the same WRAN for the duration of a superframe. In the example reported above $M$ has been fixed equal to 3. To choose the WRAN clusters for the transmission, a greedy algorithm is implemented.

In the above example, at first the coordinator schedules the transmission on the channelA, giving the transmission possibility to the WRAN which needs more among WRAN1, WRAN4, and WRAN6. To explain the assignment process, we suppose the probability vector equal to $[1 / 4,1 / 4,1 / 6,1 / 6,1 / 12,1 / 12]$. Since $p_{1}$ is greater than $p_{4}$ and $p_{6}$, the resource is assigned to the WRAN1 and $s_{-} v$ becomes $[1,0,0,0,0,0]$. Successively channelB has to be allocated, and the choice is among WRAN2, WRAN3, WRAN5. Considering that $p_{2}$ is greater than $p_{3}$ and $p_{5}, s_{-} v$ becomes $[1,1,0,0,0,0]$. At the end there is the assignment process for the channelC. The leader has to computes all the possible s_vs, according to the WRAN clusters allowed on the channelC, and it computes all the corresponding $y$ values, as illustrated in the following table. Note that the initial $\mathrm{s} v$ in this step is $[1$, $1,0,0,0,0]$, which is the resulting vector of the former step, i.e. the scheduling process of the transmission on the channelB. The step by step algorithm implementation for the channelC access scheduling is illustrated in Figure 4.

Each row of the column named "s_v" in Figure 4 shows a possible final $s \_v$ for the superframe. These vectors are used to compute the result of Equation (2), and finally, the vector which gives the highest result is chosen. The result marked with red color, namely 0.5776 , corresponds to the cluster chosen by the coordinator. The final $s_{-} v$ is $[1,1,1,1,0,0]$.

For the following superframe the coordinator restarts with the same assignment process, using as inputs the last $s \_v$, keeping unvaried the other parameters. This process is illustrated in the flow chart of Figure 6.

In Figure $\mathbf{5}$ is illustrated the WRAN access to the

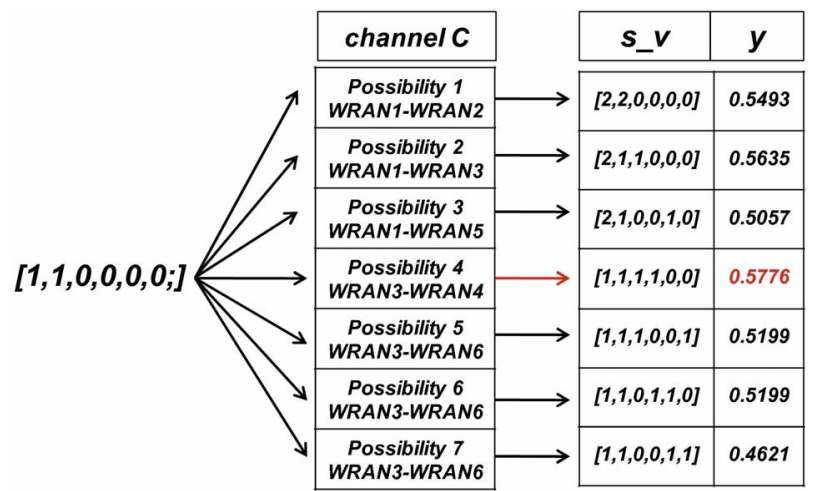

Figure 4. Assignment of the channelC.

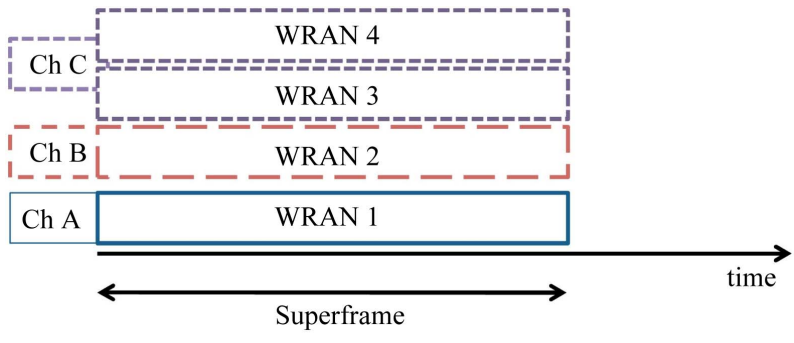

Figure 5. Channel access map for the first superframe.

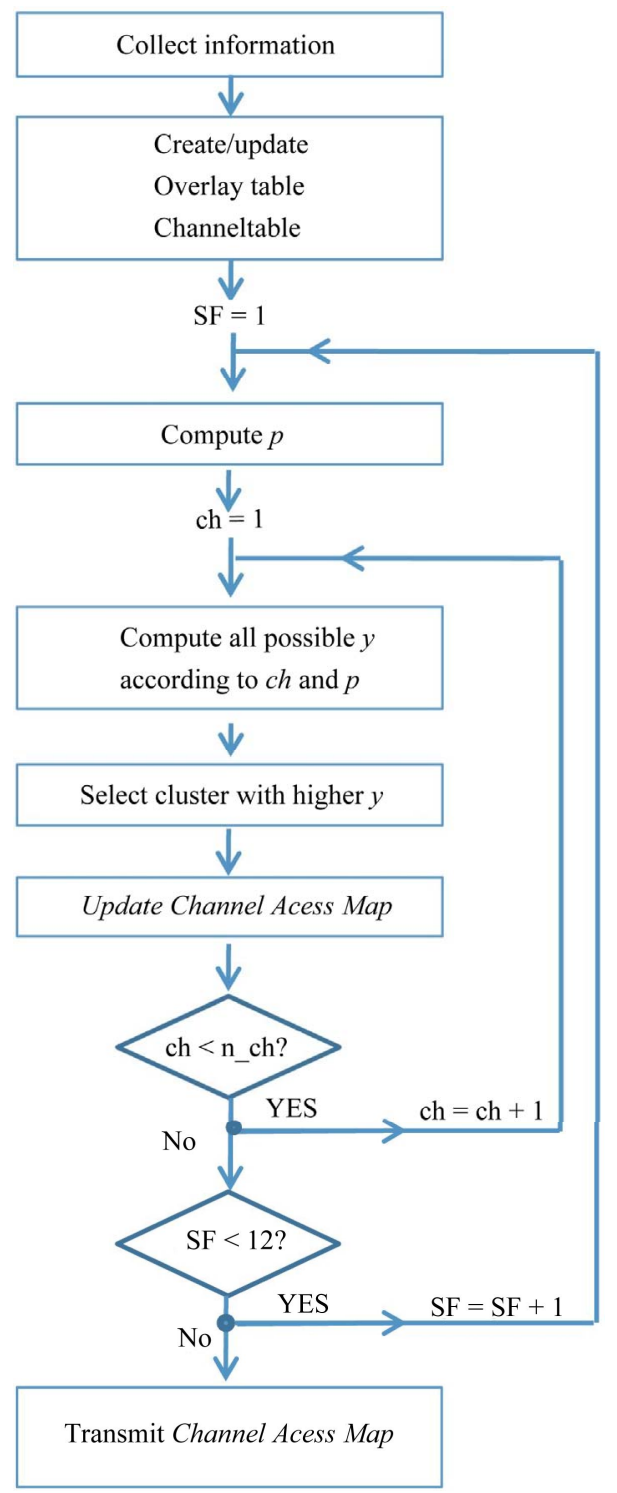

Figure 6. Example of the channel assignment.

channels during the first superframe of the assignment period.

In Figure $6 S F$ is the abbreviation of superframe, ch of channel, and $n \_c h$ is the number of available channels. As explained in Section 2, the allocation process takes no more than 12 superframes. After this, the leader discloses 
to whole WRAN community the channel access map. The $s \_v$ is reset to the initial value, which is $[0,0,0,0,0$, $0]$, and the allocation operations start again with a new sensing phase.

\subsection{Considerations on the Function y}

The function $y$ was build as in Equation (2) because it increases when the resources are assigned to the WRANs with higher requests: they have higher $p_{i}$, so increasing the corresponding $n_{i}$ the global value of $y$ will increase too. Moreover y has a logarithmic growth, for this reason $y$ raises very fast with the first assigned superframes to the WRANs with higher $p_{i}$ s. Subsequently, in the sum, the contributes of these will raise more slowly, because it is a logarithmic function, then, for increasing $y$, it is necessary to assign superframes to the WRANs with lower $p_{i} \mathrm{~s}$.

Now we prove that, according to the chosen criteria, the optimal solution is obtained when $n_{i}=n p_{i}$, where $n$ is the total number of assigned superframes. This means that, in case of $p_{i}$ all equal among them, our criteria chooses the $s \_v$ where $n_{i}$ approaches more to the mean value $\tilde{n}=n / N$. In general, in the optimal solution $n_{i}$ is proportional to the related $p_{i}$, i.e. respects the resource request of WRANi.

Our criterion aims at maximizing the following sum:

$$
\sum_{i=1}^{N} p_{i} \ln \left(n_{i}+1\right)
$$

with $n_{i} \geq 0$. If $n_{i}$ is equal to 0 , any contribution is given to the sum. For this reason we will next consider $n_{i} \geq 1$. To show the optimality condition the above quantity can be rewritten in the following way by summing and subtracting the same term:

$$
\sum_{i=1}^{N} p_{i}\left[\ln \left(n_{i}+1\right)+\ln n_{i}-\ln n_{i}\right]
$$

After few steps we obtain:

$$
\sum_{i=1}^{N} p_{i} \ln n_{i}+\sum_{i=1}^{N} p_{i} \ln \frac{n_{i}+1}{n_{i}}
$$

It is possible to observe that the following inequality is always true:

$$
\sum_{i=1}^{N} p_{i} \ln \frac{n_{i}+1}{n_{i}} \leq 1
$$

In particular, the above sum has limit 0 when $n_{i}$ increases. The above sum is negligible and consequently:

$$
\sum_{i=1}^{N} p_{i} \ln \left(n_{i}+1\right) \approx \sum_{i=1}^{N} p_{i} \ln \left(n_{i}\right)
$$

Let us now prove the following inequality:

$$
\sum_{i=1}^{N} p_{i} \ln \left(n_{i}\right) \leq \sum_{i=1}^{N} p_{i} \ln \left(n p_{i}\right)
$$

By drawing everything to the fist member we obtain:

$$
\sum_{i=1}^{N} p_{i} \ln \left(n_{i}\right)-\sum_{i=1}^{N} p_{i} \ln \left(n p_{i}\right) \leq 0
$$

Given that the first member can be written as:

$$
\sum_{i=1}^{N} p_{i} \ln \frac{n_{i}}{n p_{i}}
$$

and that [19] ln $y \leq y-1$, we obtain:

$$
\sum_{i=1}^{N} p_{i} \ln \frac{n_{i}}{n p_{i}} \leq \sum_{i=1}^{N} p_{i}\left[\frac{n_{i}}{n p_{i}}-1\right]
$$

Considering that the second member of the inequality

$$
\sum_{i=1}^{N} p_{i}\left[\frac{n_{i}}{n p_{i}}-1\right]=\sum_{i=1}^{N} p_{i} \frac{n_{i}}{n p_{i}}-\sum_{i=1}^{N} p_{i}
$$

and that:

$$
\sum_{i=1}^{N} p_{i} \frac{n_{i}}{n p_{i}}-\sum_{i=1}^{N} p_{i}=0
$$

we proved that:

$$
\sum_{i=1}^{N} p_{i} \ln \left(n_{i}\right) \leq \sum_{i=1}^{N} p_{i} \ln \left(n p_{i}\right)
$$

which means that first and second member become equal when $n_{i}=n p_{i}$. We can conclude that $\sum_{i=1}^{N} p_{i} \cdot \ln \left(n_{i}\right)$ is maximized when $n_{i}=n p_{i}$. With reference to Equation (14), we can assert in the same way that $\sum_{i=1}^{N} p_{i} \cdot \ln \left(n_{i}+1\right)$ is maximized when $n_{i}=n p_{i}$.

By applying the criteria exposed in this Section, our algorithm assigns resources to WRANs in such a way to satisfy the required QoS. In fact we proved that the resources guaranteed to each WRAN depend on the desired transmission probability $p_{i}$.

To create the channel allocation map the coordinator evaluates all possible $s \_v$ s for each superframe; for each potential $s \_v$ the community leader calculates the result of Equation (3), and finally chooses the state which returns the greatest results. According to this decision the channel access map is created and the $s \_v$ is the input for the follow step of the algorithm to decide the allocation for the subsequent superframe.

\section{Performance Evaluation}

In this Section the performance are evaluated. At this aim a simulation tool was developed to reproduce the functionality of the introduced resource sharing method. Moreover, to appraise the performance of the proposed scheme the simulation results are compared with other ones obtained according to solutions already known in 
literature. The comparison has been made with Max_Min [10] method, and On Demand Spectrum Contention (ODSC) protocol [8]. According to the Max_Min method, after computing the chromatic number, and the group of WRANs which can utilize the same channel, the available spectrum is divided according to the principle of Max-Min fairness criterion. Assuming that a graph is $x$ colorable the total available band, namely $F$, is divided into $x$ parts; so $F / x$ is the band assigned to each group of WRANs with the same color in the graph. If the WRAN cluster needs less then $F / x$, the excess is redistributed to the remaining group of WRANs. The Max_Min method has a centralized structure. In the ODSC protocol the WRANs accede to the channels with a contention mechanism. The contention decisions are made by the coexisting networks in a distributed way, without the necessity of a central coordinator.

Furthermore, a target of the proposed scheme is to realize a fair resource sharing, to demonstrate that this goal is reached a fairness index is introduced. In particular the Jain's index [20] is evaluated for a set of scenarios of different topology.

Initially we show the results obtained in relation to particular network topology. In the simulations we suppose a topology like one shown in Figure 3, where the community is composed by 6 WRANs. As explained in the last Section the assignment process has to last 12 superframes. The WRANs communicate to the coordinator of the total amount of data that their CPEs need to transmit. This data are collected in a vector, namely load: the $i^{\text {th }}$ element of the vector reports the amount of data to be transmitted in the WRANi.

Considering the spectral efficiency of the exploited modulation and the bandwidth of the usable channels, the coordinator estimates how many superframes are required by the WRANs, to satisfy the demands. These are the WRAN requests, and are stored in a vector, namely request: the $i^{\text {th }}$ element of the vector returns the number of superframes requested by the WRANi.

In the simulations we supposed a transmission mode supported in IEEE 802.22 [18], in particular we referred to a QPSK modulation.

Figure 7 shows simulation results computed according to these parameters:

- The number of available channels is 2 ;

- The bandwidth is $6 \mathrm{MHz}$, which is the width of a television channel;

- QPSK modulation with spectral efficiency of 1.01;

- The frame duration is $10 \mathrm{~ms}$;

- The simulation time, fixed to 12 superframes, is equivalent to $1.92 \mathrm{~s}$;

- The load vector is $[6.7,6.7,9.6,9.6,11.5,11.5] \mathrm{Mbit}$;

- The request vector corresponds to the load is $[7,7,10$, $10,12,12]$ superframes.

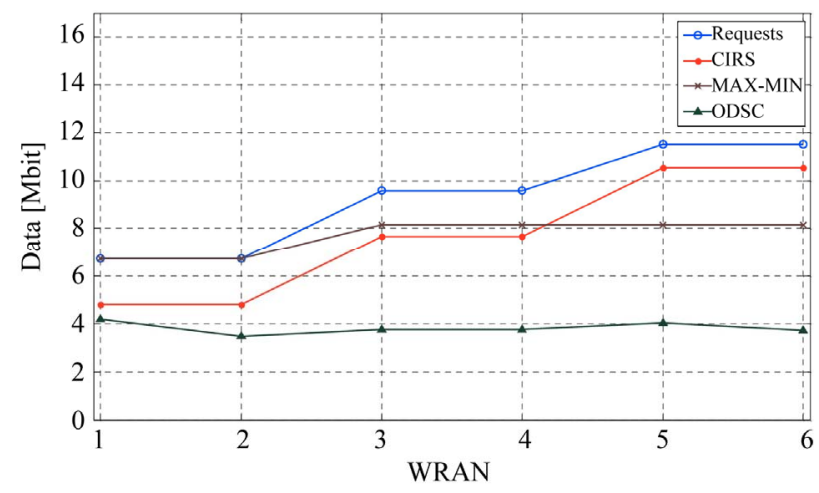

Figure 7. Example of resource sharing.

At first we assume that all the WRANs are able to hear all the channels. In the sequel we will release this hypothesis, and we will show how the allocation process results change.

According to the above listed value set, simulations were run, the results are reported in Figure 7. The numerical results compare the amount of data which each WRAN needs to transmit and the amount of data actually transmitted. In Figure 7, 4 curves are shown which are: the WRAN requests, and the amount of transmitted data utilizing CIRS, ODSC and Max_Min methods. The amount of data is expressed in megabits. The WRAN requests, marked by the symbol "o", as indicated in the list, are supposed increasing. The simulation results prove that in this context the ODSC is the less suitable method. The price to pay for the advantage of not needing a coordinator is the high collision probability. With the ODSC method there is a wastage of resources because it is afflicted by the interference problem. Moreover, this allocation policy does not take into account the WRAN requests, so the $r$ sources are allocated on the basis of the contention mechanism. Otherwise Max_Min method, as the CIRS, needs a coordinator and the resource division in made according to the WRAN requests. However the Max_Min does not assign the resource proportionately with the community member requests.

In the exposed example the chromatic number is 3 , and the corresponding 3 clusters are: WRAN1-WRAN2, WRAN3-WRAN4, and WRAN5-WRAN6. The total available band is $12 \mathrm{Mhz}$, which is the result of two TV channels. To each cluster a third of the total band is assigned, which is $4 \mathrm{MHz}$. The total amount of data which is possible to transmit could be computed as follow:

$$
\text { Data = Bandwidth.time-spectral_efficiency; }
$$

where in the example the time, i.e. the transmission period is $1.92 \mathrm{~s}$, the spectral efficiency is 1.01 and the bandwidth is equal to $4 \mathrm{Mhz}$. The amount of data that can be send are $7.75 \mathrm{Mbit}$. WRAN1 and WRAN2 need to transmit 6.7 Mbit, so $4 \mathrm{MHz}$ band is more than the first cluster needs. The excessive resources are divided among 
the other clusters. However the resources assigned to the second and the third clusters are not sufficient to satisfy their requests. Though the Max_Min method takes into account the necessity of the members, to avoid wastage, i.e. some WRANs have more they need. The simulation shows that, although WRAN3, WRAN4, WRAN5 and WRAN6 have different demands they obtain the same resources, while, as shown in Figure 7 the curve which depicts the assigned resource according to the CIRS scheme has the same trend of the curve which represents the requests. Even if the available resources are not sufficient to satisfy all the WRAN demands, the available band is divided proportionally to the demands.

Other simulations were run considering the same scenario, but chancing the WRAN requests: results are shown in Figure 8.

The CIRS scheduling does not satisfy only the WRAN2 requests, while the other demands are satisfied. For the Max_Min scheduling, the formed clusters are 3, the same of the previous example, to which is assigned a bandwidth of the $4 \mathrm{MHz}$. This bandwidth is overabundant for the cluster composed by WRAN5-WRAN6, so the surplus is divided among the other clusters. The bandwidth assigned to these is not sufficient to satisfy their requests. In particular note that the resources assigned to the second cluster, WRAN3-WRAN4, are more then WRAN4 needed and less then WRAN3 required. But WRAN3 and WRAN4 are included in the same cluster so a resource redistribution is not possible. The same happened for the WRANs of the first cluster. This example is introduced to demonstrate that the Max_Min scheduling tends to be inefficient because there is a band wastage for some WRANs, which have more than they need, while the resources are not sufficient for other members.

Figure 8 proves that the red curve, representing CIRS scheduling, follows the trend of the request curve. Otherwise the Max_Min scheduling assigns the same resources to WRANs which do not have the same band requirements. In the last simulation set the spatial diversity was introduced. In this condition to apply the Max_Min method is not possible, because this method is based on the hypotheses that all the channels have the same coverage area. The cause is that to compute the chromatic number all the WRANs have to hear the same channels. For this reason we can compare only ODSC with CIRS. The supposed channel availability is the same indicated in Table 2. We supposed 3 available channels, including the channelC, available for all the WRANs, while the other two channels are visible only to a subset of WRANs. The other simulation parameters are the same previously used. The numerical results are shown in Figure 9. As already illustrated the protocol ODSC does not allow an efficient resource sharing, even if the ODSC is able to manage the spatial diversity condition. The CIRS scheme allows a

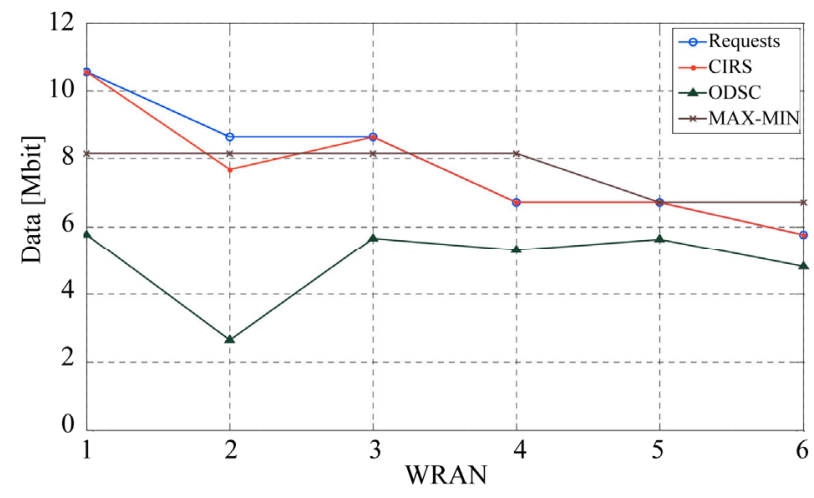

Figure 8. Example of resource sharing.

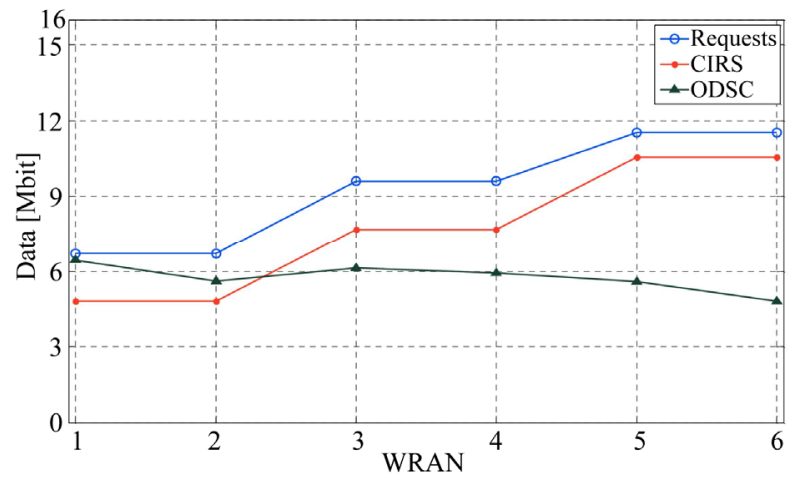

Figure 9. Resource sharing with spatial diversity.

proportional division of available resources.

In the follow some results are shown obtained computting the Jain's fairness index [20]. A fairness index $(F I)$ is a real number that measures how fair or unfair the resources are shared among the competitors. The Jain's index is calculated as:

$$
F I=\frac{\left|\sum_{i=1}^{N} x_{i}\right|^{2}}{N \cdot \sum_{i=1}^{N} x_{i}^{2}}
$$

where $N$ is the total number of applicants resources, while $x_{i}$ is defined as:

$$
x_{i}= \begin{cases}\frac{\text { all }_{i}}{r e q_{i}} & \text { if } \text { all }_{i}>r e q_{i} \\ 1 & \text { otherwise }\end{cases}
$$

where $a l l_{i}$ and $r e q_{i}$ are respectively the allocated and required resources of the user . $_{\text {. }}$

In Figure 10 shows each value illustrates the mean fairness indexes of 50 simulations. For each group of 50 simulations the WRAN number $\left(N_{-W R A N}\right)$ has been fixed, while the number of available channels $\left(n_{-c h}\right)$, the network topology and the channel coverage area and the WRAN requests were randomly varied. The randomization of the network topology was obtained randomly 


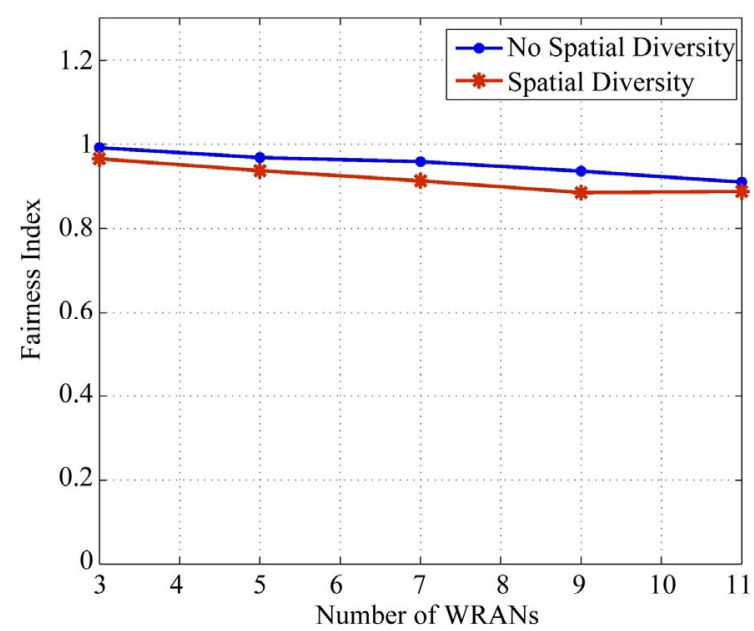

Figure 10. Fairness indexes.

generating the overlapping table. It is a symmetric square matrix, with $N_{\text {WRAN }}$ size, composed only by elements equal to 1 or 0 , and with diagonal elements all equal to 1 . The variable $n_{-c h}$ is in the range [2,5].

For each scenario a simulation was run, and the fairness index is computed according to the Equation (16). The results are collected to compute the mean values, reported in Figure 10. Moreover, two types of simulation sets are created, with or without spatial diversity. Chosen $n_{-c h}$, the channel coverage area is randomly generated, creating a channel table randomly.

The channel table has size equal to $N_{\text {WRAN }} \cdot n_{-c h}$, and the elements are casually chosen between 0 or 1 .

In the Figure 10 the symbols "o" marks the values depicting the simulations where the channel coverage area is supposed uniform.

The FI is always greater then 0.88 this means that the allocation scheme is a fair method. Note that the fairness index increases releasing the assumption of spatial diversity. This happens because the index is independent of the amount of shared resources but it depends on how they are distributed in the network. This is connected with the spatial diversity and the distribution of resources, which are not available for all competitors in the same way.

The indexes are all high, because the CIRS scheme adopts a model for resource distribution which does not disadvantage the WRANs which have a reduce accessibility to the channels.

\section{Conclusions}

In this paper a resource sharing algorithm was presented, which allows an optimized distribution of resources among 802.22 networks.

The WRANs, arranged in a community, elect the coordinator which is responsible to collect information, to implement the algorithm for resource sharing, and to divulge the transmission map to all members of the community.

The resource management is based on the channel reuse. The assigned time unit is the superframe; this allows to schedule the transmission for long time periods, without excessively increasing the computational complexity.

Furthermore, the algorithm is able to take into account the WRAN QoS requirements giving more bandwidth for a longer time to WRANs with higher demand. The channel allocation process regards, as well as the QoS requirements, the spatial diversity, i.e. channels with different coverage area and then WRANs with different channel availability.

A simulation tool was build to test the resource sharing method. Performance evaluation, shown in Section 4, proved that the introduced algorithm achieves a band assignment proportional to the WRAN requests. Moreover the results prove that the introduced scheme is significantly better than other methods as schemes based on the graph theory, or decentralized solutions because it increases the spectral efficiency. Moreover the fairness of the allocation process was demonstrated with the help of the Jain's fairness index.

\section{REFERENCES}

[1] C. Passiatore and P. Camarda, "A Centralized InterNetwork Resource Sharing (CIRS) Scheme in IEEE 802.22 Cognitive Networks," The 10th IEEE IFIP Annual Mediterranean Ad Hoc Networking Workshop, Favignana Island, 12-15 June 2011, pp. 17-24.

[2] P. Camarda, C. Cormio and C. Passiatore, "An Exclusive Selfcoexistence (ESC) Resource Sharing Algorithm for Cognitive 802.22 Networks," 5th IEEE International Symposium on Wireless Pervasive Computing (ISWPC), Modena, 5-7 May 2010, pp. 128-133.

[3] K. Ben Letaief and W. Zhang, "Cooperative Communications for Cognitive Radio Networks," Proceedings of the IEEE, Vol. 97, No. 5, 2009, pp. 878-893. doi:10.1109/JPROC.2009.2015716

[4] I. F. Akyildiz, W.-Y. Lee, M. C. Vuran and S. Mohanty, "Next Generation/Dynamic Spectrum Access/Cognitive Radio Wireless Networks: A Survey," Computer Networks, Vol. 50, No. 13, 2006, pp. 2127-2159. doi:10.1016/j.comnet.2006.05.001

[5] R. Tandra, M. Mishra and A. Sahai, "Extended Edition: What Is a Spectrum Hole and What Does It Take to Recognize One?” University of California, Berkeley, 2008.

[6] K. Bian and J.-M. Park, "A Coexistence-Aware Spectrum Sharing Protocol for 802.22 WRANs," The 18th International Conference on Computer Communications and Networks, San Francisco, 3-6 August 2009, pp. 1-6.

[7] H. Wendong, D. Willkomm, M. Abusubaih, J. Gross, G. Vlantis, M. Gerla and A. Wolisz, "Cognitive Radios for Dynamic Spectrum Access-Dynamic Frequency Hopping 
Communities for Efficient IEEE 802.22 Operation," Communications Magazine, Vol. 45, No. 5, 2007, pp. 80-87.

[8] H. Wendong, M. Gerla, G. Vlantis and G. Pottie, "Efficient, Flexible, and Scalable Inter-Network Spectrum Sharing and Communications in Cognitive IEEE 802.22 Networks," 1st International Workshop on Cognitive Radio and Advanced Spectrum Management, Aalborg, 14 February 2007, pp. 1-5.

[9] S. Sengupta, S. Brahma, M. Chatterjee and S. Shankar, "Enhancements to Cognitive Radio Based IEEE 802.22 Air-Interface," IEEE International Conference on Communications, Glasgow, 24-28 June 2007, pp. 5155-5160. doi:10.1109/ICC.2007.852

[10] Z. Fan and R. Zhang, "Spectrum Allocation and Medium Access in Cognitive Radio Wireless Networks," Wireless Conference (European), Aalborg, 17-20 May 2009, pp. 90-95.

[11] F. Kelly, "Charging and Rate Control for Elastic Traffic," European Transactions on Telecommunications, Vol. 8, No. 1, 1997, pp. 33-37. doi:10.1002/ett.4460080106

[12] C. Cordeiro, K. Challapali, D. Birru and S. Shankar, "IEEE 802.22: The First Worldwide Wireless Standard Based on Cognitive Radios," 1st IEEE International Symposium on New Frontiers in Dynamic Spectrum Access Networks, Baltimore, 8-11 November 2005, pp. 328-337.

[13] C. Sacchi and F. Granelli, "Qos-Driven Radio Resource Allocation for OFDMA Networks Based on a Game Theoretical Approach," Electrical and Electronic Engineering, Vol. 21, No. 2, 2010, pp. 283-290.
[14] C. Cordeiro, K. Challapali, et al., "IEEE 802.22: An Introduction to the First Wireless Standard Based on Cognitive Radios," Journal of Communications, Vol. 1, 2006, pp. 38-47.

[15] C. Cormio and K. Chowdhury, "A Survey on Mac Protocols for Cognitive Radio Networks," Ad Hoc Networks, Vol. 7, No. 7, 2009, pp. 1315-1329.

[16] S. Hwang, J. S. Um, M. Song, C. Kim, H. Park and Y. Kim, "Design and Verification of IEEE 802.22 WRAN Physical Layer," 3rd International Conference on Cognitive Radio Oriented Wireless Networks and Communication, Singapore, 15-17 May 2008, pp. 1-6.

[17] A. Sharifloo, M. Mirakhorli, M. Esmaeili and A. T. Haghighat, "A Leader Election Algorithm for Clustered Groups," International Conference on Industrial and Information Systems, Penadeniya, 9-11 August 2007, pp. 14.

[18] C. Stevenson, G. Chouinard, Z. Lei, W. Hu, S. Shellhammer and W. Caldwell, "IEEE 802.22: The First Cognitive Radio Wireless Regional Area Network Standard," Communications Magazine, Vol. 47, No. 1, 2009, pp. 130-138. doi:10.1109/MCOM.2009.4752688

[19] S. Benedetto, E. Biglieri and V. Castellani, "Digital Transmission Theory," Prentice Hall, Upper Saddle River, 1987.

[20] R. Jain, D. Chiu and W. Hawe, “A Quantitative Measure of Fairness and Discrimination for Resource Allocation in Shared Computer Systems," DEC Research, Technical Report, 1984. 